\title{
Lifestyle modifications to attenuate the exaggerated blood pressure response to exercise in men
}

\author{
Takeshi Otsuki ${ }^{1, *}$ \\ ${ }^{1}$ Faculty of Sport and Health Sciences, Ryutsu Keizai University, 120 Ryugasaki, 301-8555 Ibaraki, Japan
}

*Correspondence: takeshi.otsuki17@gmail.com (Takeshi Otsuki)

\begin{abstract}
The blood pressure response to physical activities is an essential contributor to ambulatory blood pressure and a risk factor for future cardiovascular disease. Peak exercise blood pressure and the blood pressure elevation from rest to peak exercise are higher in apparently healthy men without a prior history of hypertension than in their female peers. Lifestyle modifications can decrease blood pressure during aerobic and resistance exercise. However, there may be sex differences in the effects of lifestyle modifications on blood pressure responses to exercise. Additionally, the optimal blood pressure interventions probably differ between men and women due to sex differences in lifestyles. In men, hypertension not only increases the risk of cardiovascular disease but also worsens quality of life by contributing to erectile dysfunction. Further studies are warranted to attenuate the exaggerated blood pressure response to exercise in men.
\end{abstract}

\section{Keywords}

Aerobic exercise; Dietary habit; Dietary supplementation; Exercise blood pressure; Exercise training; Resistance exercise; Sex difference

The prevalence of hypertension differs by sex; specifically, a higher proportion of young and middle-aged men have hypertension compared with age-matched women. The National Health and Nutrition Examination Survey 20132016 in the United States reported that the prevalences of hypertension in men and women, respectively, were $26 \%$ and $13 \%$ in those aged $20-34$ years, $43 \%$ and $32 \%$ in those aged $35-44$ years, $56 \%$ and $50 \%$ in those aged $45-54$ years, and $66 \%$ and $65 \%$ in those aged $55-64$ years [1]. The proportion of men with hypertension further increases after age 65 (71\% in those $65-74$ years and $80 \%$ in those 75 years and older), even though it is lower than in women of the same age (78\% and $86 \%$, respectively) [1]. Consistent with the link between hypertension and mortality, age-adjusted death rates attributable to hypertension were higher in men relative to women [1].

Adults with normal resting blood pressure should still pay attention to ambulatory blood pressure (ABP). Banegas et al. [2] reported that masked hypertension (i.e., normal office blood pressures and elevated 24-hour ABP) had a larger hazard ratio for all-cause mortality versus sustained hyperten- sion. An essential contributor to ABP is the blood pressure response to physical activities, since an exaggerated response to aerobic exercise, such as walking or cycling, is a risk factor for future cardiovascular disease [3]. Additionally, resistance exercise, such as lifting objects and mopping, is a component of many activities of daily living. Elevated blood pressure during resistance exercise is a risk factor for elevated 24-hour $\mathrm{ABP}$, independent of resting blood pressure [4]. It is also a marker of masked hypertension [5] and a risk factor for future essential hypertension [6].

The role of exercise blood pressure as a risk factor for cardiovascular disease may be more crucial in men than in women. Daida et al. [7] demonstrated that peak exercise blood pressure and the blood pressure elevation from rest to peak exercise (i.e., delta blood pressure) were higher in apparently healthy men without a prior history of hypertension than in their female peers. The sex differences in peak and delta blood pressure during exercise persisted in individuals aged 70-79 years [7]. Lifestyle modifications to attenuate the exaggerated blood pressure response to exercise is a significant research issue, particularly in men. 
Regarding blood pressure during aerobic exercise, several excellent studies have investigated the effects of lifestyle modifications. Kokkinos et al. [8] and Pitsavos et al. [9] demonstrated that 16 weeks of aerobic exercise training decreased blood pressure during submaximal and peak exercise in normotensive and hypertensive men. Kim et al. [10] reported that blood pressure during submaximal ergometer cycling decreased 2 hours after the intake of grape seed extract in prehypertensive men. These results suggest that exercise training and dietary supplementation decrease blood pressure during aerobic exercise in men. Additionally, Michishita et al. [11] showed that a 12-week lifestyle modification program consisting of aerobic exercise and diet counseling decreased blood pressure elevation during ergometer cycling in women with an exaggerated blood pressure response to exercise.

With respect to blood pressure during resistance exercise, Kruse et al. [12] reported that 8 weeks of sodium nitrate supplementation decreased mean blood pressure during hand grip exercise in men and women with peripheral artery disease. In healthy middle-aged and older men and women, we demonstrated that 6 weeks of aerobic exercise training decreased systolic blood pressure during arm curl exercise [13]. The aerobic training-induced reduction in blood pressure during resistance exercise may be associated with improved nitric oxide bioavailability [14]. These results suggest that lifestyle modifications can decrease the blood pressure response to resistance exercise.

However, there may be sex differences in the effects of lifestyle modifications on blood pressure responses to exercise. For example, Collier et al. [15] reported that arterial stiffness, a crucial factor contributing to increased blood pressure during aerobic [16] and resistance [4, 5] exercise, was increased in men but not in women during 4-week resistance exercise training. Resistance exercise is an essential training modality to prevent frailty. If resistance training increases the blood pressure response to exercise in men, measures including aerobic exercise training, dietary modifications, and supplementation may be needed. Regarding another potential difference between men and women, Hayashi et al. [17] demonstrated that estrogen receptor-alpha polymorphism affects arterial stiffness in postmenopausal women who engaged in daily physical activity. If polymorphisms were implicated in the blood pressure response to exercise in men, this would help guide decisions regarding lifestyle modifications. Finally, lifestyles differ between men and women. For instance, Japanese men are more likely to be smokers and to have a higher sodium intake than women. Thus, the optimal blood pressure interventions probably differ between men and women. The beneficial effects of sexspecific lifestyle modifications can be detected in implementation and epidemiological studies, even if sex differences are not observed in well-controlled intervention studies with small sample sizes.

In men, hypertension not only increases the risk of cardiovascular disease but also worsens quality of life by contributing to erectile dysfunction. Further studies are warranted to attenuate the exaggerated blood pressure response to exercise in men.

\section{Author contributions}

TO is the sole contributor to this article.

\section{Funding}

This work was supported by JSPS KAKENHI Grant Number 20K11311.

\section{Conflict of interest}

The author declare that no conflicts of interest exist.

\section{References}

[1] Virani SS, Alonso A, Benjamin EJ, Bittencourt MS, Callaway CW, Carson AP, et al. Heart disease and stroke Statistics-2020 update: a report from the American Heart Association. Circulation. 2020; 141: e139-e596.

[2] Banegas JR, Ruilope LM, de la Sierra A, Vinyoles E, Gorostidi M, de la Cruz JJ, et al. Relationship between clinic and ambulatory blood-pressure measurements and mortality. New England Journal of Medicine. 2018; 378: 1509-1520.

[3] Keller K, Stelzer K, Ostad MA, Post F. Impact of exaggerated blood pressure response in normotensive individuals on future hypertension and prognosis: systematic review according to PRISMA guideline. Advances in Medical Sciences. 2017; 62: 317-329.

[4] Otsuki T, Kotato T. Blood pressure during resistance exercise is associated with 24-h ambulatory blood pressure and arterial stiffness. Journal of Physical Fitness and Sports Medicine. 2019; 8: 209-216.

[5] Koletsos N, Dipla K, Triantafyllou A, Gkaliagkousi E, Sachpekidis V, Zafeiridis A, et al. A brief submaximal isometric exercise test 'unmasks' systolic and diastolic masked hypertension. Journal of Hypertension. 2019; 37: 710-719.

[6] Chaney RH, Eyman RK. Blood pressure at rest and during maximal dynamic and isometric exercise as predictors of systemic hypertension. American Journal of Cardiology. 1988; 62: 1058-1061.

[7] Daida H, Allison TG, Squires RW, Miller TD, Gau GT. Peak exercise blood pressure stratified by age and gender in apparently healthy subjects. Mayo Clinic Proceedings. 1996; 71: 445-452.

[8] Kokkinos PF, Narayan P, Fletcher RD, Tsagadopoulos D, Papademetriou V. Effects of aerobic training on exaggerated blood pressure response to exercise in African-Americans with severe systemic hypertension treated with indapamide +/- verapamil +/enalapril. American Journal of Cardiology. 1997; 79: 1424-1426.

[9] Pitsavos C, Chrysohoou C, Koutroumbi M, Aggeli C, Kourlaba G, Panagiotakos $\mathrm{D}$, et al. The impact of moderate aerobic physical training on left ventricular mass, exercise capacity and blood pressure response during treadmill testing in borderline and mildly hypertensive males. Hellenic Journal of Cardiology. 2011; 52: 6-14.

[10] Kim J, Kim K, Choi H, Park S, Stebbins CL. Grape seed extract supplementation attenuates the blood pressure response to exercise in prehypertensive men. Journal of Medicinal Food. 2018; 21: 445-453.

[11] Michishita R, Ohta M, Ikeda M, Jiang Y, Yamato H. Effects of lifestyle modification on an exaggerated blood pressure response to exercise in normotensive females. American Journal of Hypertension. 2017; 30: 999-1007.

[12] Kruse NT, Ueda K, Hughes WE, Casey DP. Eight weeks of nitrate supplementation improves blood flow and reduces the exaggerated pressor response during forearm exercise in peripheral artery disease. American Journal of Physiology. Heart and Circulatory Physiology. 2018; 315: H101-H108.

[13] Otsuki T, Kotato T, Zempo-Miyaki A. Habitual exercise decreases systolic blood pressure during low-intensity resistance exercise in 
healthy middle-aged and older individuals. American Journal of Physiology. Heart and Circulatory Physiology. 2016; 311: H1024H1030.

[14] Otsuki T, Nakamura F, Zempo-Miyaki A. Nitric oxide and decreases in resistance exercise blood pressure with aerobic exercise training in older individuals. Frontiers in Physiology. 2019; 10: 1204.

[15] Collier SR, Frechette V, Sandberg K, Schafer P, Ji H, Smulyan H, et al. Sex differences in resting hemodynamics and arterial stiffness following 4 weeks of resistance versus aerobic exercise training in individuals with pre-hypertension to stage 1 hypertension. Biology of
Sex Differences. 2011; 2: 9

[16] Otsuki T, Maeda S, Kesen Y, Yokoyama N, Tanabe T, Sugawara J, et al. Age-related reduction of systemic arterial compliance induces excessive myocardial oxygen consumption during sub-maximal exercise. Hypertension Research. 2006; 29: 65-73.

[17] Hayashi K, Maeda S, Iemitsu M, Otsuki T, Sugawara J, Tanabe T, et al. Estrogen receptor-alpha genotype affects exercise-related reduction of arterial stiffness. Medicine and Science in Sports and Exercise. 2008; 40: 252-257. 